\title{
Problems Encountered in Seated Arm Reach Posture Reconstruction: Need for a More Realistic Spine and Upper Limb Kinematic Model
}

\author{
Xuguang Wang \\ Université de Lyon, F-69622, Lyon, France, \\ INRETS, UMR_T9406, LBMC, Bron, \\ Université Lyon 1, Villeurbanne \\ xuguang.wang@inrets.fr
}

\begin{abstract}
In this paper, we will present the main problems encountered for reconstructing in-vehicle reach postures. Among the 2176 successfully captured movements, about $7.4 \%$ of them were considered as "bad quality" with a high residual error between reconstructed and measured marker positions. They mainly correspond to the far targets and in the direction that one has to elevate the arm. The results of the present study strongly suggest that a more realistic kinematic model of the upper body including the shoulder complex, pelvis and spine is required. In addition, the natural coordination between joint axes should also be used for compensating the lack of information in case of underconstraint situation and for correcting the uncertainty of surface markers positions.
\end{abstract}

Keywords: Reach, Digital human, Motion reconstruction, Motion capture, Discomfort.

\section{Introduction}

Evaluating reach capacity and difficulty is one of main requirements for ergonomic design of workplace [4-6]. Recently, we proposed a unified data based approach which aimed at predicting both reach envelopes and reach discomfort for a digital human model [18-21]. In this approach, four reach surfaces, from half-flexed arm distance to maximum reach with torso participation, need to be predicted at first from an existing database of reach postures. Then, the discomfort of a target reach is evaluated in terms of target position with respect to these four reach envelops. We applied this approach for predicting in-car driver's reach envelopes and discomfort. One of critical issues in this approach was to build-up a database of reach postures covering a large arm reachable space.

For data collecting, 2184 reach movements (twenty-four differently sized male and female subjects x 91 targets located in a large reachable space) were captured. The aim of this paper is to present the difficulties encountered in reconstructing these reach postures using a digital human model and to suggest future research directions for improving the quality of reconstructed movements. 


\section{Reach Motion Data Collecting}

Thirteen female and eleven male subjects participated in the experiment. They were selected according to three groups by stature

- Short

- Average-height

- Tall
$:<1625 \mathrm{~mm}$

: $1625-1755 \mathrm{~mm}$

:> $1755 \mathrm{~mm}$

An experimental driving mock-up was built up. It was composed of a seat, two pedals (acceleration and braking) and a steering wheel. It corresponded to a standard Renault car configuration. The subjects were asked to naturally grasp a sphere of 40 $\mathrm{mm}$ in diameter with three fingers (thumb, index and major) of the right hand from a standard driving posture. The reach posture was maintained during about 3 seconds before returning back to the starting posture. The buttock was not allowed to leave the seat. The left hand was maintained on the steering-wheel during the whole movement.
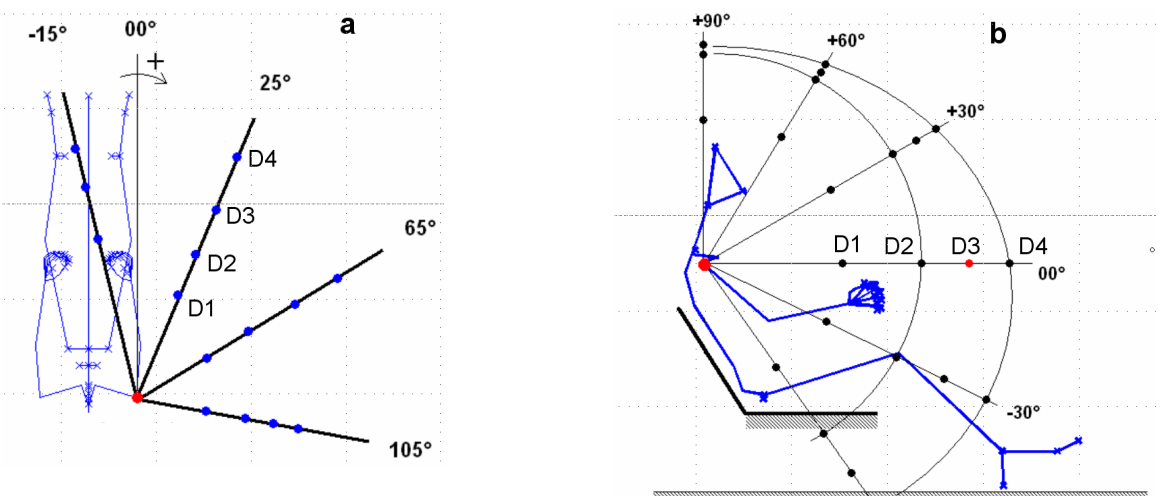

Fig. 1. Target location

The targets were located in four azimuth planes (P-15 to $\mathrm{P} 105),-15^{\circ}$ to $105^{\circ}$ from the sagittal plane passing through the right shoulder (Fig. 1a). For each azimuth plane (Fig. 1b), the targets were positioned in six elevations (E-60 to E90) (from $-60^{\circ}$ to $90^{\circ}$ ) and four distances from the shoulder (D1 to D4). The four distances were defined with respect to the maximum reach distances without (D2) and with (D4) torso movement for each elevation. D3 was the mid distance between D2 and D4. D1 was defined as the two-third of D2. Maximum reach distances D2 and D4 for each target elevation in each orientation plane were determined prior to the experiment for each subject.

In all, 75 different target locations were tested for each subject:

- 60 locations for the planes P25, P65 and P105

- 4 distances (D1, D2, D3, D4)

- 5 elevations $\left(-60^{\circ},-30^{\circ}, 0^{\circ}, 30^{\circ}\right.$ and $\left.60^{\circ}\right)$ 
- 11 locations for plane P-15

- 4 distances (D1, D2, D3, D4)

- 3 elevations $\left(0^{\circ}, 30^{\circ}, 60^{\circ}\right)$. The elevations $-30^{\circ}$ and $-60^{\circ}$ were eliminated because of the interference between the body and the seat.

- The target E0-D1 in the plane P-15 was also eliminated because of the interference with the steering-wheel.

- 4 locations for the pole (elevation $90^{\circ}$ )

- 4 distances (D1, D2, D3, D4)

In order to test the repeatability of discomfort rating and reach posture, the target E0-D3 for each azimuth plane was repeated two times. In addition, two different grasping types were also tested for the same target location in order to look at the effects of grasping type of reach discomfort. Therefore, each subject carried out 91 $(75+8+8)$ reach movements in total. Movements were captured using the optoelectronic system VICON with 10 cameras at a frequency of $50 \mathrm{~Hz}$. The detailed description of the experimental procedure for data collecting can be found in Wang et al. [21].

\section{Posture Reconstruction Method}

Motion reconstruction consists in calculating the joint angles of a human model from captured marker trajectories, called also inverse kinematics motion reconstruction. Several model-based motion reconstruction methods have been proposed in the past. An up-to-date review of motion reconstruction methods can be found in Ausejo and Wang [2]. For instance, Lu and O'Connor [13] proposed a Global Optimization Method (GOM), searching for an optimal posture minimizing, in the least square sense, the overall distances between the measured and the model-determined marker coordinates, of all the body segments at each frame. In a recent European research project named REALMAN, Ausejo and his colleagues [19-1] proposed a similar approach using natural coordinates and applied it to the whole body motion reconstruction.

In the present study, the inverse kinematics solver is based on generalized pseudoinverse as initially proposed by Liegois [12]:

$$
\Delta \theta=J^{+} \Delta X+\left(I-J^{+} J\right)\left(\theta-\theta_{r e f}\right)
$$

where $\Delta X$ is the incremental marker displacement, $\Delta \theta$ the joint angle change. $J$ is called the Jacobian and relates linearly $\Delta X$ and $\Delta \theta . J^{+}$is the pseudo inverse of $J$. $\theta$ is the joint angle array representing the body posture and $\theta_{r e f}$ is a reference posture. The first term of this equation minimizes the distance between the measured and the model-determined marker coordinates and is of high priority. The second term tends to bring the actual posture to a reference one within the range of solutions admitted by the first term. $\left(I-J^{+} J\right)$ is the projection operator of $\left(\theta-\theta_{\text {ref }}\right)$ into the null space of $J$. The second term is of lower priority. This formulation therefore enables 
the driving of over-constrained as well as under-constrained chains: most frequently, two or more markers are placed on each segment of the four limbs. For instance, the arm posture is therefore over-constrained and not dependant on the reference posture. On the other hand, the spine, where it is frequently not possible to place markers, is driven by markers on the pelvis and on the upper torso. The spine here is underconstrained. An infinite number of solutions can exist, which minimize the marker distances. The second term of the equation therefore attracts the posture to a reference one, leading to a unique solution. Otherwise, without the second term, a solution will be found, which is approaching to its initial guess.

This inverse kinematics algorithm is implemented in the motion reconstruction and simulation software RPx (see Monnier et al., [15] for a general presentation of RPx), which is used in this study for reach posture reconstruction. Several preparatory steps are necessary before joint angle calculation:

1. Definition of the digital twin of a real subject from twenty-four measured anthropometric dimensions. For this, the tool named BodyBuilder by the software editor Human Solutions is used.

2. Verification and fine adjustment of the digital human model created in the step 1 . It consists in a visual inspection by superimposing the model with different views of a reference posture (Fig. 2). RPx allows one to adjust segment length and joint angle.

3. Marker attachment. Once the human model is superimposed correctly with the photos, each marker is attached to one body segment in order to obtain its local coordinates in the corresponding body segment coordinate system.

4. Joint angle calculation by minimizing the distance model-based marker positions and those measured by the motion capture system.

In order to reconstruct the posture of the spine and pelvis, the standard seated driving posture is used as the reference posture. In addition, an empirical coordination laws of spine joint joints are also used (Monnier et al) [14], allowing a better control of spine joint angles by four torso global attitude parameters (axial rotation, forward and backward flexion-extension, lateral flexion, compression/elevation). Fig.3 shows an example of reconstructed reach posture.
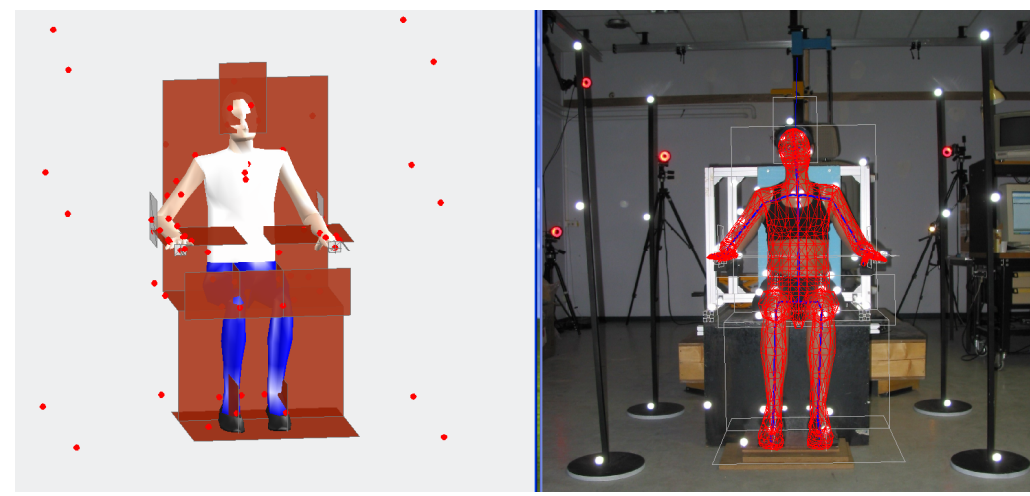

Fig. 2. Verification and fine adjustment of model's dimensions and marker attachment 


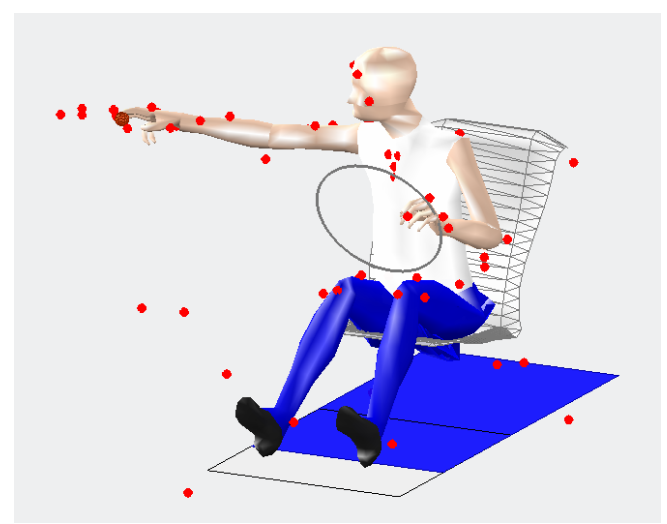

Fig. 3. An example of reconstructed reach posture for the target P065-E0-D3

\section{Evaluation of Reconstructed Reach Postures}

In order to evaluate the quality of reconstructed reach postures, we calculated the mean value $(\mathrm{m})$ of the residual errors of the following eight markers and their standard deviation (s) for each posture:

- Markers on the right (lfe_r) and left knee (lfe_l)

- Marker on the chest (sn)

- Markers on the right upper limb: shoulder (ac_r), elbow (lhe_r), wrist (ms_r)

- Markers on the hand : hand back (smc_r), index fingertip (index_r)

It is well known that the soft tissue artefact (STA) is the main error source for motion analysis [11]. For instance, Fuller et al. [18] reported that skin-mounted markers on the lower limb (thigh and shank segments) could exhibit displacements with respect to the underlying bone of up to $20 \mathrm{~mm}$. Moreover, the procedure used for defining an individualized 3-D geometric model is quite approximate. In a past study [19], we compared the seven anthropometric dimensions between direct measurements and those obtained by superimposing a digital model with a least 2 photos of different views in two different reference postures (standing and sitting). Their average differences could reach as high as $28.7 \mathrm{~mm}$ for the upper arm length in the standing posture and $44 \mathrm{~mm}$ for the sitting height for the sitting posture. Considering possible large relative movement between surface marker and the underlying bone as well as the approximation made for individualized digital twin of the subject, the reconstructed posture is qualified "bad quality" if both $\mathrm{m}$ and s are greater than $20 \mathrm{~mm}$. Using this criteria, the postures with a high residual error but uniformly distributed $(\mathrm{m}>20 \mathrm{~mm}$ and $\mathrm{s}<20 \mathrm{~mm})$ and those with a large dispersion of residual errors but a small global residual error $(\mathrm{m}<20 \mathrm{~mm}$ and $\mathrm{s}>20 \mathrm{~mm})$ are considered as "correctly" reconstructed". In the first case, the residual errors are relatively uniformly distributed among the selected markers, and corresponding reconstructed postures were visually natural looking in general. In the second case, the residual errors are unevenly distributed with a low average error. It may be due to high error of one or two mislabelled 
Table 1. Number of badly reconstructed postures according to target distance, plane, and elevation

\begin{tabular}{|l|l|l|l|l|l|}
\hline \multicolumn{2}{|c|}{ Distance } & \multicolumn{2}{c|}{ Plane } & \multicolumn{2}{c|}{ Elevation } \\
\hline D1 & 11 & P-15 & 56 & E-60 & 2 \\
\hline D2 & 33 & P25 & 21 & E-30 & 5 \\
\hline D3 & 47 & P65 & 22 & E0 & 16 \\
\hline D4 & 71 & P105 & 63 & E30 & 29 \\
\hline & & & & E60 & 72 \\
\hline & & & & E90 & 38 \\
\hline
\end{tabular}

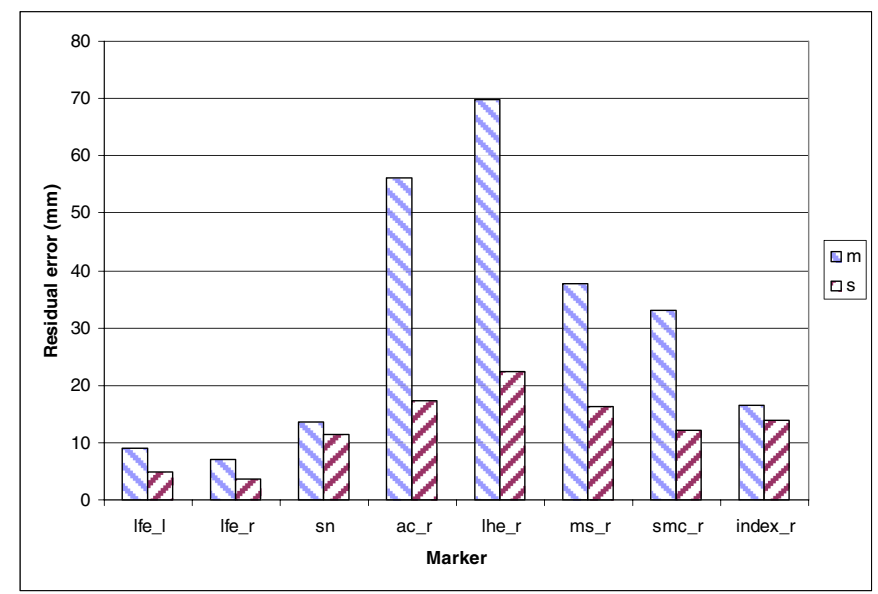

Fig. 4. Means and standard deviations of the residual errors of the eight selected markers of the 162 movements qualified "badly reconstructed"

markers. As the method is a global optimisation procedure, it is quite robust to the missing and mislabelling of a small number of markers.

Among the 2176 (24 subjects x 91 targets -8 unsuccessfully captured trials) captured reach movements, 162 postures are considered "badly reconstructed", representing $7.4 \%$. Their distribution according to target location is summarized in Table 1 . One can see that these badly reconstructed postures mainly corresponded to the targets located at far distances (D3 and D4) in the planes $-15^{\circ}$ and $105^{\circ}$ with a high arm elevation (azimuth $60^{\circ}$ and $90^{\circ}$ ).

Fig. 4 shows the distribution of residual errors for the eight selected markers. One can see that the main errors are located at the shoulder (ac_r), elbow (lhe_r) and wrist (ms_r). The marker shoulder (ac_r) is in the area of high skin movement when the arm is elevated. For far targets, the elbow is almost fully extended, leading to the alignment of the upper arm and forearm and thus to high uncertainty about their axial rotation. In addition to skin movement artefact, this may partly explain high residual errors for the markers elbow (lhe_r) and wrist (ms_r). However, the residual errors decrease for more distal markers (smc_r, index_r). This may lead to unrealistic hand posture. 


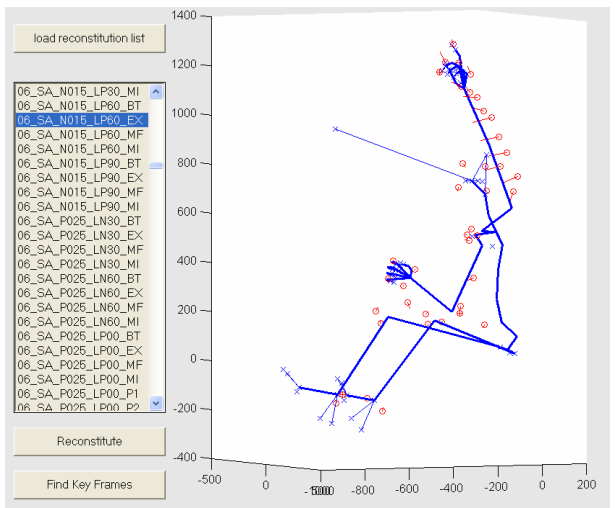

Fig. 5. Example of a "badly" reconstructed posture for the target $\mathrm{P}(-15)-\mathrm{E} 60-\mathrm{D} 4$, showing high residual error of the markers attached at the upper and lower arms. The residual error of a marker and its direction is represented by the red stick from the marker.
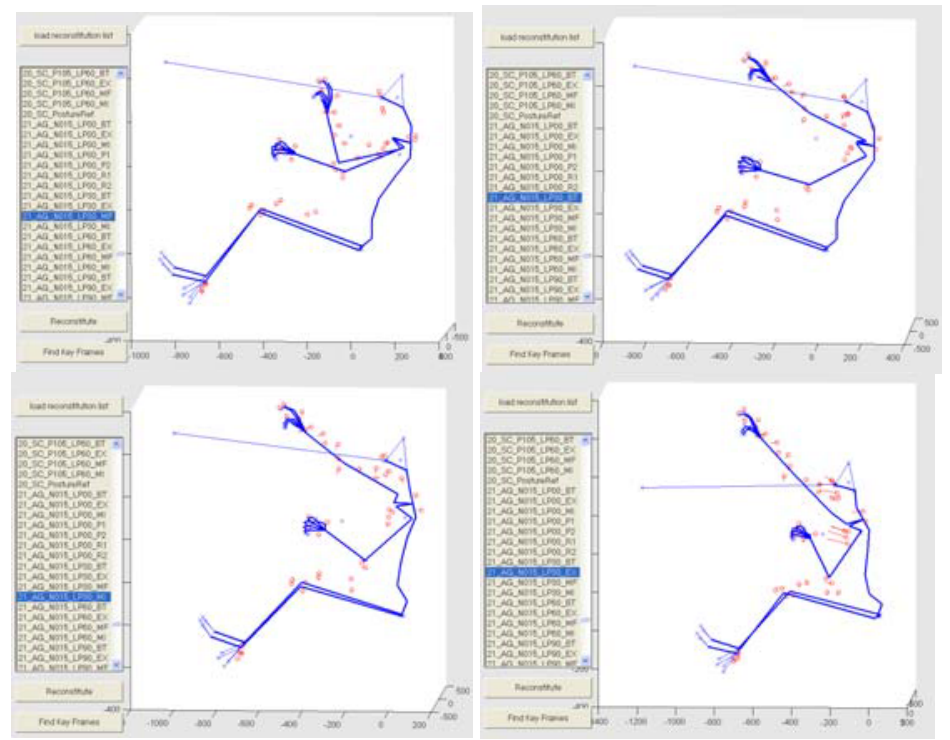

Fig. 6. An example of "badly" reconstructed reach posture for the target $\mathrm{P}(-15)-\mathrm{E} 30-\mathrm{D} 4$. The postures of the three other reach distances in the same reach direction are also shown. The residual error of a marker and its direction is represented by the red stick from the marker.

Figures 5 and 6 show two examples of "badly" reconstructed postures, one illustrating high residual errors of the markers attached on the upper limb, the other for the thorax. 


\section{Concluding Remarks}

In this paper, we have illustrated the main problems encountered in reconstructing invehicle reach postures. Among the successfully captured movements, about $7.4 \%$ of them were considered as "bad quality" with a high error between reconstructed and measured marker positions. They mainly correspond to the far targets in the direction that one has to elevate the arm. The markers attached on the elbow had the highest residual errors, and then came the markers at the shoulder. The residual errors decrease for distal markers at the wrist, the hand back and the fingertips. The fingertips being the last kinematic chain of the upper body, this may imply that the joint angles are probably not correctly estimated for these targets.

The kinematic human model used in this paper is based on the RAMSIS model, which is widely used in automotive industries for car interior design. Like most of digital human models, the spine is represented by six spherical joints. The shoulder complex is simplified as an open chain composed of one spherical joint at the glenohumeral joint and one revolute joint at the sterno-clavicular joint. The results of the present study clearly show that this simplified model is probably good enough for the targets close to the body without high arm elevation. But for far and high targets, such a simplified model may not be appropriate.

In this study, the 3-D individualised human model is created based on classical 1-D anthropometric dimensions. Frequently, some manual adjustments are required to fit the photos. Using this procedure, the advantage is that the internal structure of human model (joint centre, joint axes) is identified implicitly. However, we do not know how good the identified internal structure is with respect to the real anatomic structure. Clearly we need information which allows the identification of internal kinematic structure from external measurable parameters, such as palpable anatomic bony landmarks.

There is also a need to define natural coordination laws of joint axes for compensating the lack of information in case of under-constraint situation and for correcting the uncertainty of surface markers positions. In fact, for far targets, one has to fully extend the arm and to move the trunk. When the elbow is fully extended, the upper and lower arms are almost aligned; it is difficult to correctly estimate their axial rotations due to high uncertainty of the marker positions at the elbow and wrist. In a seated position, it is difficult to put many makers on the trunk. In our study, only four markers were attached at the thoracic cage and two markers at the pelvis. The movement of the trunk and pelvis was clearly under-constraint in our case. It is also true for the shoulder movement. Only one marker was attached at the shoulder. Due to the skin movement artefact, the movement of the sterno-clavicular joint is difficult to be estimated.

The difficulties encountered in arm reach posture reconstruction for far and elevated targets strongly suggest that a more realistic kinematic model of the upper body including the shoulder complex, pelvis and spine is required. In addition, the natural coordination between joint axes should also be used in case of underconstraint situation and for correcting the uncertainty of surface markers positions. 


\section{Acknowledgement}

This study is partly supported by the car manufacturer Renault. The author would like to acknowledge Gilles Monnier for his technical assistance as well as Nicolas Chevalot, Sebatien Parello, Julien Causse for data collecting.

\section{References}

1. Ausejo, S., Suescun, Á., Celigüeta, J., Wang, X.: Robust Human Motion Reconstruction in the Presence of Missing Markers and the Absence of Markers for Some Body Segments. In: SAE International conference and exposition of Digital Human Modeling for Design and Engineering, Lyon, France, July 4-6 (2006) SAE paper 2006-01-2321

2. Ausejo, S., Wang, X.: Motion Capture and Reconstruction. In: Duffy, V.G. (ed.) Handbook of Digital Human Modeling. Taylor \& Francis Group, Abington (2008)

3. Boydstun, L.E., Kessel, D.S.: Maximum reach models for the seated pilot. Center for ergonomics, college of engineering, University of Michigan (March 1980)

4. Chaffee, J.W.: Methods for determinating driver reach capability. SAE report 6901205 , New York (1969)

5. Chaffin, D.B., Faraway, J.J., Zhang, X., Woolley, C.: Stature age and gender effects on reach motion postures. Human factors 42(3), 408-420 (2000)

6. Chateauroux, E., Wang, X.: Effects of age, gender and target location on seated reach capacity and posture. Human Factor 50(2), 211-226 (2008)

7. Chevalot, N., Wang, X.: Experimental investigation of the discomfort of arm reaching movements in a seated position. SAE Transactions, Journal of Aerospace 1, 270-276 (2004)

8. Fuller, J., Liu, L.J., Murphy, M.C., Mann, R.W.: A comparison of lowerextremity skeletal kinematics measured using skin- and pin-mounted markers. Hum. Mov. Sci. 16, 219-242 (1997)

9. Jung, E.S., Choe, J.: Human reach posture prediction based on psychophysical discomfort. Internaltional Journal of Industrial Ergonomics 18, 173-179 (1996)

10. Kennedy, F.W.: Reached capability of men and women: A three dimensional analysis. In: Aerospace medical research laboratory, Wright-Patterson Air force base, Yellow springs, Ohio (July 1978)

11. Leardini, A., Chiari, L., Della Croce, U., Cappozzo, A.: A. Human movement analysis using stereophotogrammetry. Part 3: soft tissue artefact assessment and compensation. Gait Posture 21, 212-225 (2005)

12. Liegeois, A.: Automatic supervisory control of the configuration and behavior of multybody mechanisms. IEEE Transactions on systems, man and cybernetics 7(12), 868-871 (1977)

13. Lu, T.W., O'Connor, J.J.: Bone position estimation from skin marker co-ordinates using global optimisation with joint constraints. Journal of Biomechanics 32, 129-134 (1999)

14. Monnier, G., Wang, X., Beurier, G., Trasbot, J.: Coordination of spine degrees of freedom during a motion reconstruction process. SAE 2007 Transactions Journal of Passenger Car - Electronic and Electronic Systems (2007) SAE Paper 2007-01-2454

15. Monnier, G., Wang, X., Trasbot, J.: RPx: A motion simulation tool for car interior design. In: Duffy, V.G. (ed.) To be published in Handbook of Digital Human Modeling, Taylor \& Francis Group, Abington (2008) 
16. Reed, M.P., Parkinson, M.B., Chaffin, D.B.: A new approach to modeling driver reach. In: 2003 SAE World Congress, Detroit, Michigan, March 3-6 (2003) SAE Paper N ${ }^{\circ}$ 2003-010587

17. Sengupta, A.K., Das, B.: Maximum reach envelope for the seated and standing male and female for industrial workstation design. Ergonomics 43(9), 1390-1404 (2000)

18. Wang, X., Chateauroux, E., Chevalot, N.: A data-based modeling approach of reach capacity and discomfort for digital human models. In: Duffy, V.G. (ed.) HCII 2007 and DHM 2007. LNCS, vol. 4561, pp. 215-223. Springer, Heidelberg (2007)

19. Wang, X., Chevalot, N., Monnier, G., Ausejo, S., Suescun, Á., Celigüeta, J.: Validation of a model-based motion reconstruction method developed in the Realman project. In: SAE International conference and exposition of Digital Human Modeling for Design and Engineering, June 14-16 (2005) SAE paper 2005-01-2743

20. Wang, X., Chevalot, N., Monnier, G., Trasbot, J.: From motion capture to motion simulation: an in-vehicle reach motion database for car design. In: SAE International conference and exposition of Digital Human Modeling for Design and Engineering, Lyon, France, July 4-6 (2006) SAE Paper 2006-01-2362

21. Wang, X., Chevalot, N., Trasbot, J.: Prediction of in-vehicle reach surfaces and discomfort by digital human models. In: SAE International conference and exposition of Digital Human Modeling for Design and Engineering, Sheraton Station Square, Pittsburgh, Pennsylvania, USA, June 17-19 (2008) SAE paper $\mathrm{N}^{\circ}$ 2008-01-1869

22. Wang, X., Verriest, J.P.: A geometric algorithm to predict the arm reach posture for computer-aided ergonomic evaluation. The Journal of Visualization and Computer Animation 9, 33-47 (1998)

23. Zhang, X., Chaffin, D.B.: A three-dimensional dynamic posture prediction model for invehicle seated reaching movements: development and validation. Ergonomics 43(9), 1314-1330 (2000) 